\title{
Maduración Osea de Niños de 0 a 6 Años. Muñeca y Mano II. Parte. Análisis de Probitos para Huesos Aislados
}

\author{
Dr. Carlos Valenzuela ${ }^{1}$ y Dr. Mauricio Canals $\mathbf{L}^{2}$ - Sr. Pedro Vergara V. ${ }^{3}$ \\ Skeletal Maturity of Children Aged 0 - 6 Years. \\ Wrist and Hand. II Part. Probit Analysis for Isolated Bones.
}

\begin{abstract}
The status quo and probit analysis procedure was applied to detemine means and standard deviations of the age at which Chilean children reach the Tanner-Whitehouse stages of bone maturation of the wrist and hand. Female children mature carlier, but not uniformly for all bones. It seems that this situation is inverted in the intrauterine life, at least, for the hamate bone. Several $X^{2}$, values that yield probabilities elose to 1.0 and some of them that $y$ ield probabilities less than 0.001 show that the $\mathrm{X}^{2}$ test is not regular for thesc data.

(Key words: Bone maturation. Chilean population).
\end{abstract}

En un trabajo previo ${ }^{1}$ describimos los valores cuantitativos de la maduración ósea del niño de 0 a 6 años del Area Metropolitana Norte. Señalamos que es importante conocer el paso de los estados madurativos de los huesos de la muñeca y de la mano para comparar la arnonía de maduración de diferentes poblaciones. Los estados madurativos son variables cualitativas por lo que se clasifican como presentes o ausentes en un hueso dado de un niño particular en una edad precisa. Es necesario para su análisis el método del status quo y la subsecuence aplicación del método de probito ${ }^{2 \cdot 3}$.

\section{MUESTRA Y METODO}

La muestra ya ha sido descrita ${ }^{1}$. Cada hueso de la muñeca y mano puede presentar los estados madurativos A, B, C.... H, en que el estado A corresponde a la ausencia de punto de osificación $y$ el $\mathrm{H}$ al hueso adulto ${ }^{4}$. El niño entre 0 y 6 años no presenta todas las etapas, es raro ver algún hueso más al á del estado $G$. Para cada intervalo etario se calcula el porcentaje de individuos que presentan un determinado estado madurativo. E1

1. Departamento de Biologia Celular y Genética, Facultad de Medicina, Universidad de Chile. Casilla 70061, Santiago 7. Santiago, Chile.

2. Servicio de Radiología, Hospital San José. Santiago, Chile.

3. Departamento de Salud Pública, Facultad de Medicina, Universidad de Chile. Santiago, Chile. método de probito, incor porado a un sistema de programas estadísticos (SAS) ${ }^{\mathbf{5}}$, encuentra la edad promedio para cada estado de un hueso; para ello supone que la maduración ósea corresponde a una curva de Gauss, es decir, que en la población la proporción de individuos que alcanza un estado madurativo es bien descrita por una curva Gaussiana acumulativa. Este método da la edad a la cual el $50 \%$ de la población ha alcanzado un estado madurativo y este corresponde al promedio; la desviación típica y la prueba estadistica de bondad de ajuste Gaussiano $\left(\mathrm{X}^{2}\right)$. La nomenclatura ósea es $\mathrm{Ml}=$ metacarpiano, $\mathrm{FP}=$ falange proximal, $F M=$ falange media, $\mathrm{FD}=$ falange distal, $\mathrm{HGR}=$ hueso grande. $\mathrm{HGA}=$ hueso ganchoso, $\mathbf{P}=$ piramidal, $\mathrm{SL}=$ semilunar, $\mathrm{E}=$ escafoides, $T C=$ trapecio, $T Z D=$ trapezoide. Sólo los metacarpianos 1,3 y 5 , las falanges proximales 1,3 y 5 , las falanges medias 3 y 5 y las distales 1,3 y 5 son consideradas ${ }^{1}$.

\section{RESULTADOS}

Las Tablas 1 y 2 presentan los promedios, desviaciones típicas y probabilidades de los estados óseos que pudieron ser evaluados con el método de probito para varones y mujeres respectivamente.

1 Una explicación del método de status quo y probito se encuentra en el Apéndice. 
Tabla 1.

Promedios y Desviaciones Típicas de Edades de Maduración Osea Método de Status Quo y Probito

\begin{tabular}{|c|c|c|c|c|c|c|c|}
\hline & & Varones & $N=319$ & $G L=19$ & Mujeres & $N=336$ & $\mathrm{GL}=22$ \\
\hline HUE SO & Est. & $\begin{array}{c}\bar{x} \\
\text { Meses }\end{array}$ & $\begin{array}{c}\text { D.T. } \\
\text { Meses }\end{array}$ & $\mathrm{P}$ & $\begin{array}{c}\bar{x} \\
\text { Meses }\end{array}$ & $\begin{array}{l}\text { D.T. } \\
\text { Meses }\end{array}$ & $\mathrm{P}$ \\
\hline \multirow[t]{5}{*}{ Radio } & B & 18.02 & 8.50 & 0.99 & 11.88 & 5.80 & 0.36 \\
\hline & $\mathrm{C}$ & 21.49 & 9.66 & $<0.02$ & 15.08 & 6.61 & 0.56 \\
\hline & $\mathrm{D}$ & 34.44 & 11.66 & 0.08 & 27.19 & 8.36 & 0.96 \\
\hline & $\mathrm{E}$ & 46.73 & 12.32 & $0.3 ?$ & 38.02 & 10.45 & 0.61 \\
\hline & $\bar{F}$ & 66.95 & 12.16 & 0.95 & 56.92 & 11.80 & 0.98 \\
\hline \multirow[t]{2}{*}{ Cubito } & B & 95.32 & 34.49 & 0.85 & 82.92 & $25.5 \mathrm{I}$ & $<0.02$ \\
\hline & $\mathrm{C}$ & - & & & 84.36 & 13.05 & 0.99 \\
\hline \multirow[t]{4}{*}{$M 1$} & B & 36.43 & 11.47 & 0.42 & 23.38 & 8.14 & $<0.01$ \\
\hline & $C$ & 46.58 & 13.15 & 0.30 & 28.64 & 8.42 & $<0.01$ \\
\hline & D & 60.98 & 10.68 & 096 & 42.28 & 11.19 & $<0.01$ \\
\hline & $\mathrm{r}$ & - & & - & 77.28 & 7.20 & 0.99 \\
\hline \multirow[t]{5}{*}{ M3 } & B & 22.68 & 7.74 & 0.68 & 15.36 & 4.98 & 0.91 \\
\hline & C & 29.05 & 7.03 & 0.69 & 20.40 & 5.40 & 0.41 \\
\hline & D & 40.13 & $9.8 ?$ & 0.46 & 30.25 & 8.14 & 0.08 \\
\hline & 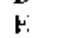 & 57.11 & 10.57 & 0.99 & 43.52 & 9.68 & 0.99 \\
\hline & $\mathrm{F}$ & & - & & 93.03 & 16.01 & 0.99 \\
\hline \multirow[t]{4}{*}{ M5 } & B & 29.48 & 8.60 & 0.71 & 18.20 & 4.92 & 0.97 \\
\hline & C. & 34.71 & 8.56 & 0.85 & 23.33 & 6.62 & 0.38 \\
\hline & D & 45.28 & 10.39 & $<0.01$ & 32.39 & 8.58 & 0.22 \\
\hline & $\mathbf{E}$ & 68.13 & 12.00 & 0.99 & 54.69 & 13.48 & 0.89 \\
\hline \multirow[t]{4}{*}{ IPP1 } & B & 36.77 & 8.79 & 0.74 & 27.16 & 8.90 & $<0.03$ \\
\hline & C & 48.79 & 11.76 & $<0.01$ & 32.76 & 10.01 & $<0.02$ \\
\hline & $\mathrm{D}$ & 54.19 & 14.49 & 0.22 & 35.57 & 10.52 & 0.44 \\
\hline & $\mathbf{E}$ & 85.55 & 24.03 & 0.99 & 60.75 & 18.82 & 0.54 \\
\hline \multirow[t]{4}{*}{ FP3 } & B & 18.80 & 6.19 & 0.99 & 11.70 & 4.94 & 0.79 \\
\hline & $\mathrm{C}$ & 20.53 & 6.04 & 0.99 & 13.99 & 5.31 & 0.89 \\
\hline & D & 27.82 & 7.10 & 0.98 & 19.43 & 5.44 & 0.73 \\
\hline & $\mathrm{E}$ & 57.99 & 15.63 & 0.29 & 38.94 & 10.27 & $<0.05$ \\
\hline \multirow[t]{4}{*}{ FP5 } & B & 27.23 & 7.89 & 0.87 & 1714 & 5.09 & 0.96 \\
\hline & $\mathrm{C}$ & 29.85 & 7.90 & 0.70 & 19.59 & 4.89 & 0.71 \\
\hline & D & 37.53 & 10.20 & 0.83 & 26.03 & 6.07 & 0.85 \\
\hline & $\mathrm{E}$ & 63.43 & 17.37 & 0.37 & 44.24 & 11.94 & 0.70 \\
\hline \multirow[t]{4}{*}{ FM3 3} & B & 27.91 & 7.55 & 0.82 & 18.85 & 6.83 & $<0.01$ \\
\hline & $C$ & 31.43 & 9.09 & 0.93 & 23,71 & 8.22 & $<0.01$ \\
\hline & $\overline{0}$ & 4420 & 10.63 & 0.15 & 31.56 & 9.04 & 0.32 \\
\hline & $E$ & 64.97 & 12.21 & 0.99 & 51.13 & 12.78 & 0.98 \\
\hline \multirow[t]{4}{*}{ FM5 } & B & 47.17 & 13.13 & 0.95 & 27.28 & 10.26 & 0.20 \\
\hline & C & 58.22 & 15.94 & 0.38 & 36.20 & 12.45 & 0.59 \\
\hline & D & 66.04 & 16.65 & $<0.01$ & 45,02 & 12.50 & 0.88 \\
\hline & E & - & - & $\cdot$. & 74.14 & 19.86 & 0.99 \\
\hline \multirow[t]{4}{*}{ FD1 } & B & 21.25 & 8.58 & 0.89 & 12.64 & 6.18 & 0.99 \\
\hline & $\mathrm{C}$ & 26.56 & 9.94 & 0.99 & 17.13 & 7.27 & 0.20 \\
\hline & D & 34.67 & 11.06 & 0.64 & 23.53 & 9.01 & $<0.01$ \\
\hline & $\mathrm{E}$ & 62.11 & 17.67 & 0.46 & 45.49 & 14.96 & $<0.01$ \\
\hline \multirow[t]{5}{*}{ FD3 } & B & 30.18 & 7.59 & 0.99 & 22.21 & 6.58 & 0.54 \\
\hline & $\mathrm{C}$ & 38.73 & 30.21 & 0.89 & 28.55 & 8.72 & 0.06 \\
\hline & $\mathbf{D}$ & 46.72 & 12.28 & 0.51 & 35.08 & 10.37 & 0.30 \\
\hline & $\mathrm{E}$ & 73.27 & 13.96 & 0.99 & 51.49 & 11.93 & 0.45 \\
\hline & $\mathrm{F}$ & - & - & -- & 72.81 & 9.74 & 0.99 \\
\hline
\end{tabular}




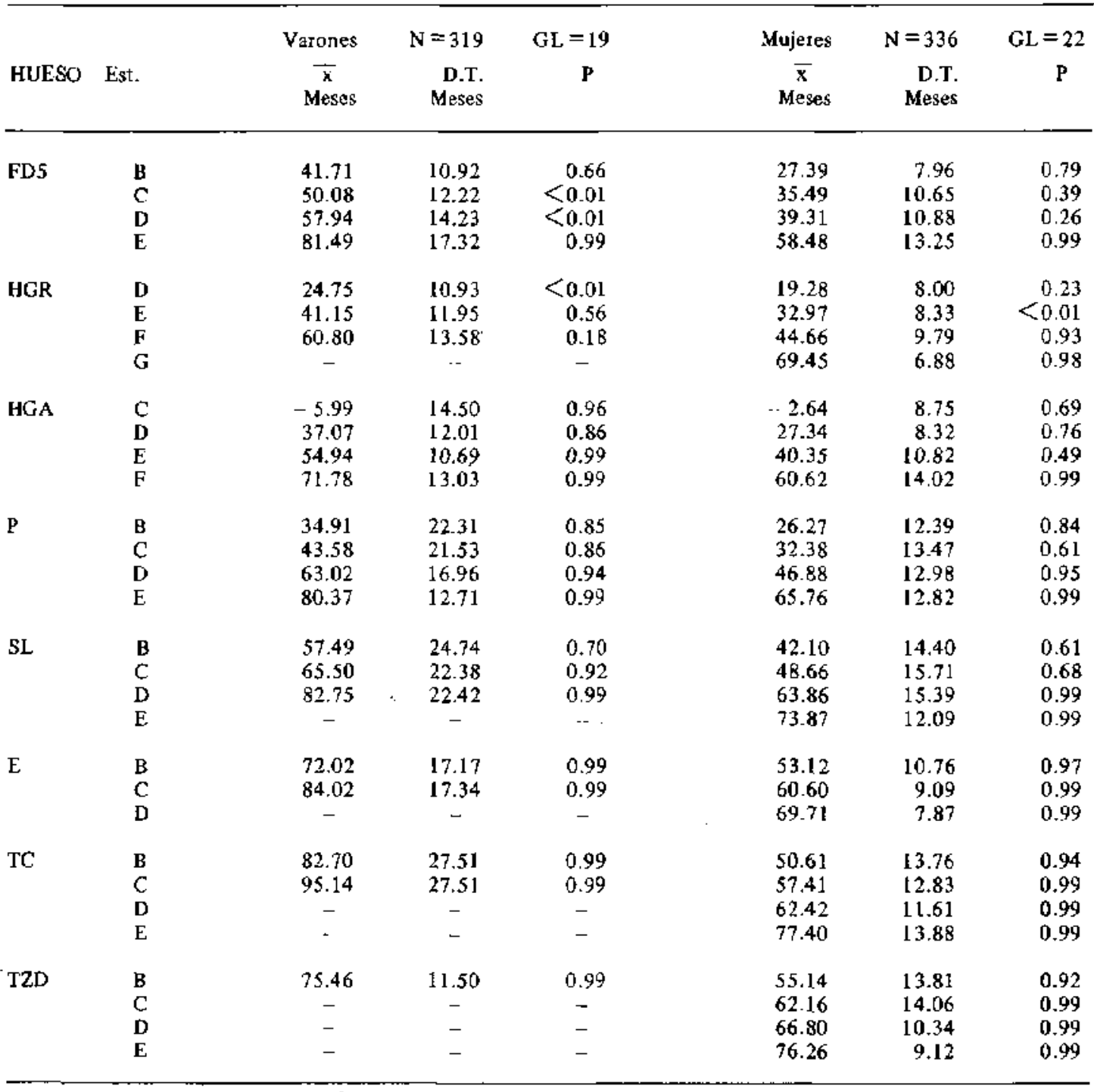

\begin{tabular}{|c|c|c|c|c|c|}
\hline $\begin{array}{l}\text { Est. } \\
\mathbf{x} \\
\mathrm{D} T\end{array}$ & $\begin{array}{l}= \\
= \\
=\end{array}$ & $\begin{array}{l}\text { Estado } \\
\text { promedio } \\
\text { Desviación tinica }\end{array}$ & $\begin{array}{l}\mathbf{P} \\
\text { GL }\end{array}$ & $=$ & $\begin{array}{l}\text { Probabilidad mayor que excepto en signo }< \\
\text { Grados de libertad }\end{array}$ \\
\hline
\end{tabular}

Las mujeres están adelantadas con respecto a los varones en todos los huesos; este adelanto se hace mayor en los estados más desartollados, se destaca excepcionalmente el hueso ganchoso en que los varones están más adelantados en el estado $\mathrm{C}$; en este caso el núme to negativo indica que el promedio o mediana se halla en la vida intrauterina. Los casos de estados no indicados o con parámetros no calculados corresponden a aquellos valores muy por fuera del intervalo etario considerado. Llama la atención, por una parte el ajuste extraordinario a la distribución Gaussiana, (Probabilidades por encima de 0.95) y por otra la existencia de lejanias a esta distribu. ción especialmente en el caso de las mujeres; cuando esta lejanía existe, ella es grande (proba. bilidad inferior a 0.01 ); es claro que estos valores de probabilidad no corresponden a un resultado aleatorio de distribuciones $\mathrm{X}^{2}$ con 19 y 22 grados de libertad respectivamente.

\section{DISCUSION}

Se confirma el adelanto marcado en madura. ción ósea de la niña respecto del niño en esta muestra. Sin embargo este adelanto no es igual en cada pieza ósea y hay evidencias de que se da la 
situación inversa en el hueso ganchoso en la vida intrauterina; esta situación está de acuerdo con el adelanto de diferenciación sexual del varón respecto de la mujer en la vida intrautcrina ${ }^{\sigma}$. La distribución de los valores de la prueba de $\mathrm{X}^{2}$ ha resultado sorprendente, no es posible explicar por simple azar tantos yalores de probabilidad tan próximos a 1.0 y varios inferiores a 0.001 (en la Tabla no aparecen porque se usan dos decimales); es posible que esta situación se deba a una limitación epistemológica que estamos estudian$\mathrm{do}^{7}$, o bien a que numerosos intervalos etarios tenian 0 ó 100\%: explicación tiene su crítica ya que habría sucedido con más uniformidad.

\section{RESUMEN}

Se uiilizó el método de status quo y probito para determinar los promedios y desviaciones típicas de la edad a la cual niños chilenos alcanzan los estados de maduración ósea de Tanner Whitehousc de la muñeca y mano. Las niñas maduran más tempranamente, pero no en forma regular para todos los huesos. Hay indicios de que el hueso ganchoso madura más tempranamente en los varones en la vida intrauterina. Hay varios valores de probabilidad de la prueba de $\mathrm{X}^{2}$ que son o muy próximos a 1.00 inferiores a 0.001 lo que es extraordinariamente irregular.

\section{AGRADECIMIENTOS}

A los Drs. Altredo Patri M., Haydée Sepúlveda B., Sonia Samith y a la Sra. Olympia Cortés por la atención de los niños y toma de radiografías. Al servicio de Salud Metropolitano Norte por las facilidades proporcionadas y al Departamento de Salud Pública de la Facultad de Medicina de la Universidad de Chilc por las unidades y materiales de computación.

\section{REFERENCLAS}

I. Canats, M.L.; Volenzuela, C.Y.; Vergaro, P.V.: Maduración ósea de niños de 0 a 6 años Muñeca $y$ mano. 1. Parte. Análisis cuantitativo. Rev. Chil. Pediatr. $56 t 5,1985$.

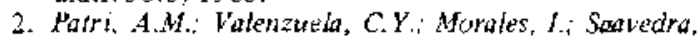

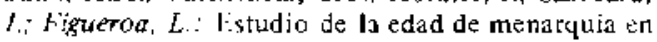
nin̉as escolares do la Enseñuza Fiscal del Sector Norte de Santilto. Cuad. Med. Soc. (Santiago). 2!, $3,1220,1980$.

3. Finney,D.J.: Statisticit Methods in Biological Assay. Sacond Edition, London - Griffin Press, 1971.

4. Tonver, J.M.; Whitehouse. R.I.: Marshall, W.A.; Healy, M.J.R.; Goldstein. H.: Assesment of skeleta] maturity and prediction of adult height (TW2 Mcthod). London Academ, Press, 1975.

5. Sas User's Guide: Statistics, 1982 Edition. Sas Institute: Inc. North Carolinu, U.S.A.. 1982.

6. Falkner, $F_{\text {.: }}$ Desarrollo Humano. Barcalona, Salvat Editores S.A.. 1969.

7. Volenzueb, C. $Y_{.:}$AJgebraic and Epistemological restrjetion in stadies on Hardy-Weinberg equilibrium. The Am. Nat. (En prensa), Mayo 1985. 